\title{
Plate-related complications after mandibular reconstruction: observational study osteotomy
}

\section{Original Article}

\author{
Dalia Radwan and Fahmy Mobarak \\ Department of Oral and Maxillofacial Surgery, Faculty of Oral and Dental Medicine, Cairo \\ University, Cairo, Egypt
}

\begin{abstract}
Background: Mandibular reconstruction following bony resection is crucial in cases of oral and mandibular tumors. Complications such as infection, plate exposure, or plate fracture can occur because of some risk factors after these reconstructive surgeries.

Objectives: The purpose of this study were to report some of the postoperative complications of reconstruction plates, and to analyze the risk factors associated with those complications.

Patients and methods: We analyzed clinical and radiological data of 19 patients who required mandibular reconstructive surgery. The complications related to plates were recorded and used as an objective measure of outcome.

Results: Plate-related complications developed in $8 / 19$ patients. The complications involved screw loosening $(n=2)$, plate fracture $(n=2)$, plate exposure $(n=3)$, and heat sensitivity $(n=1)$. No significant differences were found in plate survival rate in relation to age or sex. Moreover, complications associated with plates developed in $73.68 \%$ of the patients with defect in the mandibular body crossing the midline of the mandible. Radiotherapy decreased the survival rate of the plates, as $60 \%$ of the irradiated patients had a higher risk of plate-related complications. and angle area and $100 \%$ of the patients who had defect crossing the midline of the mandible. Radiotherapy decreased the survival rate of the plates, as $60 \%$ of the irradiated patients had a higher risk of plate-related complications.

Conclusion: The survival rate of mandibular reconstruction plate relies on mechanical and biological risk factors in terms of site of mandibular defects and radiation therapy, respectively.
\end{abstract}

Received: 20 ${ }^{\text {th }}$ November, 2017, Accepted: 20 $\frac{\text { th }}{\text { December, }} 2017$.

Key Words: mandible reconstruction, survival rate of reconstruction plates, risk factors.

Corresponding Author: Dalia Radwan, BDS, MDS, PhD, Department of Oral and Maxillofacial Surgery, Faculty of Oral and Dental Medicine, Cairo University, Cairo 11553, Egypt; Tel.: + 20122349 6188; E-mail: dalia@m-rami.org

ISSN: 2090-097X, January, Vol. 9, No. 1.

\section{INTRODUCTION}

Surgical resection of mandibular tumors often requires postresective mandibular reconstruction to recover functionality and to restore esthetic restoration of the lower face [1].

Although several techniques for mandibular reconstruction have been presented, such as soft tissue free flaps, reconstruction plates, and bone grafts, bone reconstruction is often the preferred method [2]. However, when the donor bone cannot be harvested or the lesion's prognosis is poor, bone reconstruction may not be possible $[3,4]$. In such cases, reconstruction plates can be used to restore the patient's jaw integrity, which in turn improves the patient's self-perception and self-confidence [5].
The first generation of clinically available reconstruction plates was presented in the mid-1970s. Since the early 1980 s, the standard treatment for a mandibular discontinuity defect has been the use of a rigid reconstruction plate with simultaneous or subsequent osseous reconstruction [6].

Recently, the efficacy of osteosynthesis has improved with the use of titanium reconstruction plates, which provide better biocompatibility, and locking screws for biomechanical enhancement. With these improvements, modern reconstruction plates can provide excellent anchorage and rigid fixation $[7,8]$.

Nevertheless, complications such as infection, plate exposure, fracture, or loosening of the fixation still occur occasionally [9]. Decreasing the risk of complications can be challenging, but optimizing the design and fabrication 
of reconstruction plates and improving surgical techniques may help reduce those risks [10].

Therefore, this study focused on some of the postoperative complications of reconstruction plates, and aimed to analyze the risk factors linked with those complications.

\section{PATIENTS AND METHODS}

The study included 19 patients (11 male and eight female) suffering from lesions that necessitated mandibular bony resection. They attended the Department of Oral and Maxillofacial Surgery, Faculty of Dentistry, Cairo University between 2011 and 2015. Diagnosis was confirmed by incisional biopsies before surgical resection and planned reconstructions. Twelve cases had ameloblastoma and the rest of the cases were as follows: odontogenic keratocyst (one case), Pindborg tumor (one case), and squamous cell carcinoma of the mandible (five cases). Informed consents for undergoing the surgical operation were obtained from participants according to the policy of Cairo University; moreover, the Hospital Ethical Review Board approved the study.

The surgical procedures were performed while the patients were under general anesthesia. The resultant defects were classified using HCL (Jewer's) classification [11]. In this system, $\mathrm{H}$ represents a lateral segment of any length containing a condyle and not significantly crossing the midline, $\mathrm{L}$ represents the same defect but without a condyle, and $\mathrm{C}$ represents the anterior segment between the two incisive foramina. Data for each patient including age, sex, HCL classification, and diagnosis are shown in Table 1.

The surgical protocol entailed that the resections are simultaneously coupled with placement of titanium reconstruction plates to correct the mandibular discontinuity defect and to aid in the bony reconstruction. Six months later, osseous reconstruction was done by using autogenous iliac bone grafts. We followed up the patients for another 1 year and removed the reconstruction plates by the end of the follow-up.

Table 1: Patient's demographic data, site, and diagnosis of the lesions

\begin{tabular}{|c|c|c|c|c|c|c|c|c|}
\hline \multirow[b]{2}{*}{ Patient number } & \multirow[b]{2}{*}{ Age } & \multirow[b]{2}{*}{ Sex } & \multicolumn{5}{|c|}{ Area } & \multirow[b]{2}{*}{ Pathological diagnosis } \\
\hline & & & $\mathrm{H}$ & $\mathrm{C}$ & $\mathrm{L}$ & $\mathrm{HC}$ & LC & \\
\hline 01 & 24 & Male & & & $\sqrt{ }$ & & & Ameloblastoma \\
\hline 02 & 34 & Female & & & $\sqrt{ }$ & & & Ameloblastoma \\
\hline 03 & 23 & Male & & & $\sqrt{ }$ & & & Ameloblastoma \\
\hline 04 & 26 & Female & & & $\sqrt{ }$ & & & Ameloblastoma \\
\hline 05 & 30 & Male & $\sqrt{ }$ & & & & & Ameloblastoma \\
\hline 06 & 24 & Female & & & $\sqrt{ }$ & & & Ameloblastoma \\
\hline 07 & 19 & Female & & & $\sqrt{ }$ & & & Odontogenic keratocyst \\
\hline 08 & 19 & Female & & & & & $\sqrt{ }$ & Pindborg tumor \\
\hline 09 & 58 & Male & & & & & $\sqrt{ }$ & Squamous cell carcinoma \\
\hline 10 & 21 & Female & & & $\sqrt{ }$ & & & Ameloblastoma \\
\hline 11 & 64 & Male & & & & & $\sqrt{ }$ & Squamous cell carcinoma \\
\hline 12 & 62 & Male & & & $\sqrt{ }$ & & & Squamous cell carcinoma \\
\hline 13 & 41 & Male & & & $\sqrt{ }$ & & & Ameloblastoma \\
\hline 14 & 36 & Female & & & $\sqrt{ }$ & & & Ameloblastoma \\
\hline 15 & 26 & Male & & & $\sqrt{ }$ & & & Ameloblastoma \\
\hline 16 & 67 & Male & & & $\sqrt{ }$ & & & Squamous cell carcinoma \\
\hline 17 & 25 & Female & & & & & $\sqrt{ }$ & Ameloblastoma \\
\hline 18 & 55 & Male & & & $\sqrt{ }$ & & & Ameloblastoma \\
\hline 19 & 70 & Male & & & $\sqrt{ }$ & & & Squamous cell carcinoma \\
\hline
\end{tabular}

HC, discontinuity involves the condyle, body and extends beyond the midline; LC, discontinuity involves the ramus and body and extends beyond the midline. 

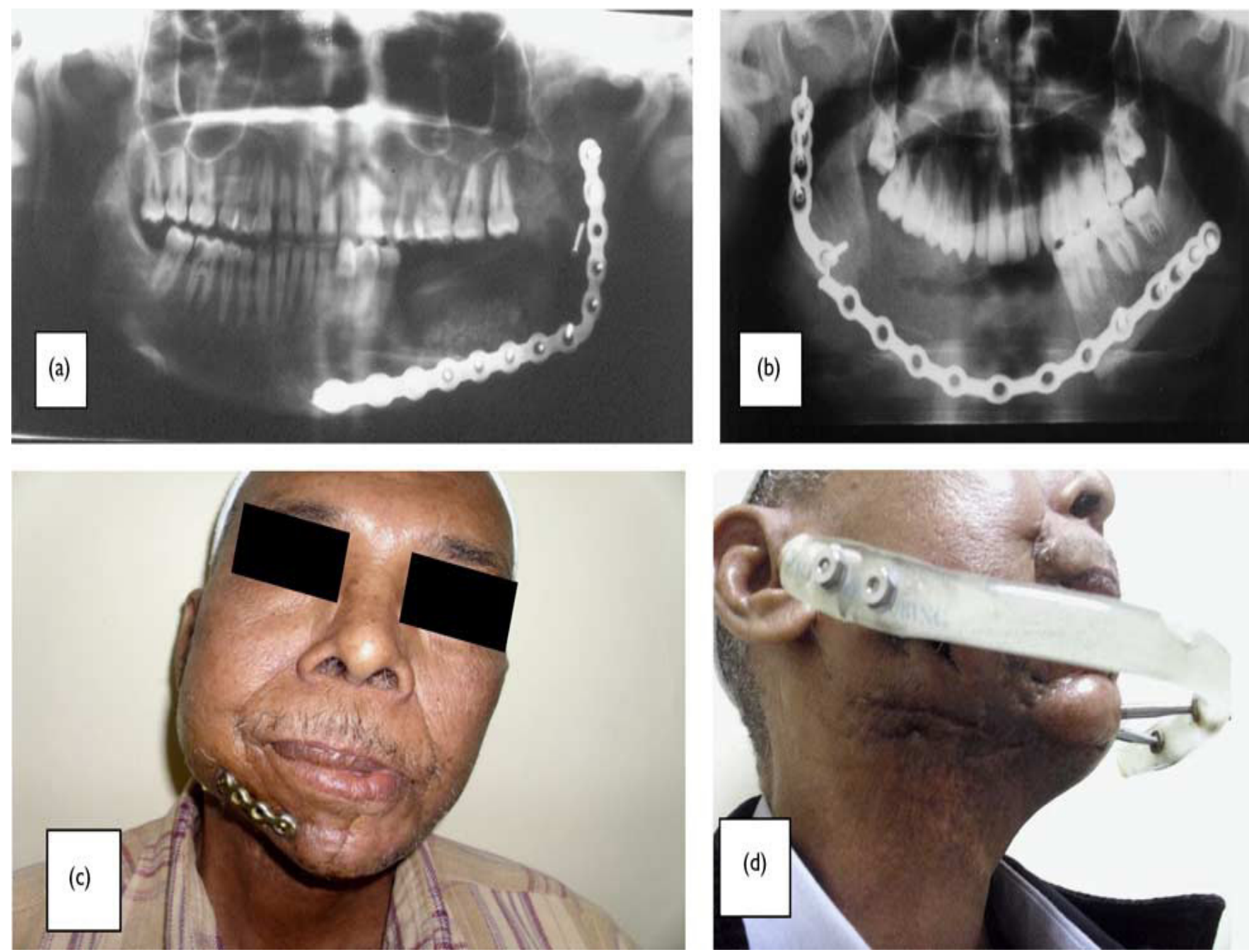

Fig. 1: Plate-related complications: (a) screw loosening, (b) plate fracture, (c) orocutaneous fistula and plate exposure, (d) application of external pin fixation after plate removal.

The complications related to plates and plate failure were recorded and used as an objective measure of outcome (Fig. 1a-c). Complications were defined as postoperative infection, screws loosening, heat sensitivity, wound dehiscence and plate exposure, or plate fracture that required plate removal.

The survival rate of the reconstruction plates was analyzed according to age, sex, site of defect, and whether postoperative radiation therapy (RT) was a risk factor. The statistical analysis for significance was tested by Pearson's $\chi 2$-test and $\mathrm{P}$ value of less than 0.05 was accepted as the level of statistical significance.

\section{RESULTS}

The mean age of the patients was 38.26 years and the mean follow-up period was $19 \pm 3$ months. The patients had a number of different diagnoses, including malignant tumors, benign tumors, and large cyst. Malignant tumors accounted for five $(26.31 \%)$ cases, benign odontogenic tumors for $13(68.42 \%)$ cases, and large odontogenic cyst for one $(5.26 \%)$ case (Table 2$)$.

Table 2: Disease processes underlying mandibular discontinuity defect cases

\begin{tabular}{lcc}
\hline \multicolumn{1}{c}{$\begin{array}{c}\text { Pathological } \\
\text { diagnosis }\end{array}$} & Cases (n) & Frequency (\%) \\
\hline Malignant tumor & 5 & 26.31 \\
Benign tumor & 13 & 68.42 \\
Odontogenic cyst & 1 & 5.26 \\
\hline
\end{tabular}

Regarding the site of the lesions, the HCL classification showed that $14(73.68 \%)$ lesions occurred in the mandibular body and angle. Four $(21.05 \%)$ lesions crossed the midline of the mandible, and one (5.26\%) lesion extended from the mandibular body to the ipsilateral condyle (Table 3). 
Table 3: Extension of the lesions based on HCL classification

\begin{tabular}{lcc}
\hline \multicolumn{1}{c}{ Site of the lesions } & Cases (n) & Frequency (\%) \\
\hline Mandibular body and angle & 14 & 73.68 \\
Lesions crossed the midline & 4 & 21.052 \\
$\begin{array}{l}\text { Lesions extended from } \\
\text { the mandibular body to } \\
\text { the ipsilateral condyle }\end{array}$ & 1 & 5.26 \\
\hline
\end{tabular}

The overall postoperative plate-related complications and affecting factors are summarized in Table 4 . Postoperative plate-related complications occurred in $42.1 \%(n=8)$ as follows: screw loosening occurred in $10.52 \%(\mathrm{n}=2)$ of the patients. Plate fracture occurred in $10.52 \%(n=2)$ of the patients, $15.78 \%(n=3)$ of the patients suffered from orocutaneous fistula associated with infection and plate exposure, and finally $5.26 \%$ $(\mathrm{n}=1)$ suffered from heat sensitivity (Fig. 2).

Table 4: Overall postoperative plate-related complications

\begin{tabular}{lcccccccc}
\hline & \multicolumn{1}{c}{ Assessments } \\
\cline { 2 - 9 } \multicolumn{1}{c}{ Variables } & $\mathrm{n} 2$ & $\mathrm{n} 8$ & $\mathrm{n} 9$ & $\mathrm{n} 11$ & $\mathrm{n} 13$ & $\mathrm{n} 15$ & $\mathrm{n} 17$ & $\mathrm{n} 19$ \\
\hline Age (years) & 34 & 19 & 58 & 64 & 41 & 26 & 25 & 70 \\
Sex & Female & Female & Male & Male & Male & Male & Female & Male \\
Diagnosis & BT & BT & MT & MT & BT & BT & BT & MT \\
HCL classification & L & LC & LC & C & L & L & LC & L \\
Postoperative RT & $\times$ & $\times$ & $\sqrt{ }$ & $\sqrt{ }$ & $\times$ & $\times$ & $\times$ & $\sqrt{ }$ \\
Complication & Plate & Heat & Plate & Plate & Screw & Plate & Plate & Screw \\
& fracture & sensitivity & exposure & exposure & loosening & fracture & exposure & loosening \\
Plate removal & $\sqrt{ }$ & $\times$ & $\sqrt{ }$ & $\sqrt{ }$ & $\times$ & $\sqrt{ }$ & $\sqrt{ }$ & $\times$ \\
\hline
\end{tabular}

BT, benign tumor; L, lateral segment of any length not containing a condyle; LC, lateral segment of any length crossing the midline; MT, malignant tumor; RT, radiation therapy.

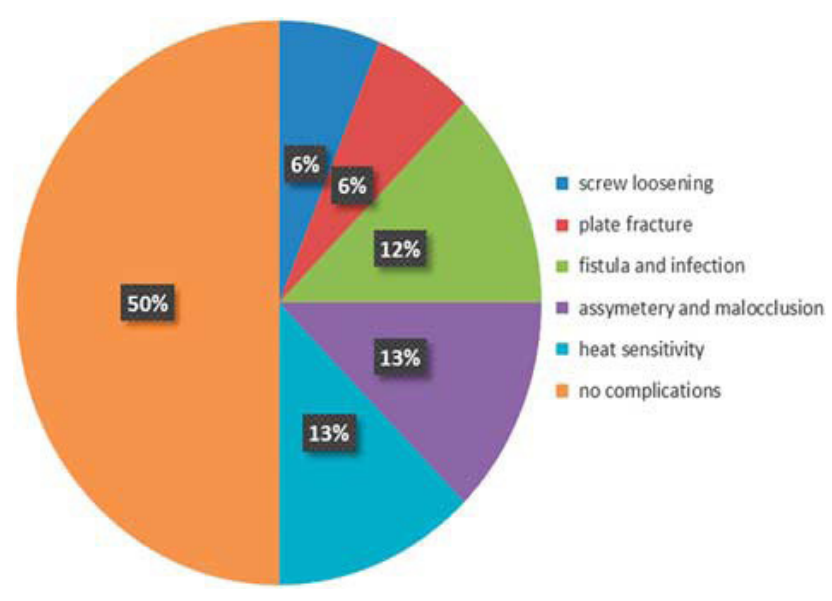

Fig. 2: Pie chart showing the percentage of postoperative platerelated complications.

Exposed reconstruction plates associated with orocutaneous fistula and infection was removed, and the patients were treated by external pin fixation (Fig. 1d). Those who experienced plate fracture were treated by replacing the current plate with another plate that was $2.7 \mathrm{~mm}$ in thickness. Patients with contour defect and those with screw loosening refused the plate removal surgery. Regarding the patient who suffered heat sensitivity, she adapted the complication 3 months later. $\chi 2$-Test revealed that the survival rate of the reconstruction plate was not significantly related to the patient's age $(P \geq 0.1)$ or sex $(P \geq 0.1)$. Complications associated with the reconstruction plate developed in $62.5 \%$ of the patients with a discontinuous defect in the mandibular body and angle area. Additionally, 100\% of the patients who had discontinuous defect crossing the midline of the mandible suffered plate-related complications. Radiotherapy decreased the survival rate of the plates, as $60 \%$ of the irradiated patients had a higher risk of platerelated complications.

\section{DISCUSSION}

The main objectives of mandibular reconstruction are to achieve functional and esthetic recovery by restoring mandibular arch continuity, to maintain soft tissue coverage, and to improve the patient's postoperative quality of life. The success rate of mandibular reconstruction has increased because of improvements in plate designing and materials. However, plate-related complications still develop frequently and can sometimes cause serious problems to patients [12].

Our findings suggest that mechanical risk factors, such as plate strain and fatigue, and biological risk factors, such as RT, affect the reconstruction plate's survival rate. 
Shibahara [13] reported that bite force upsurges the mechanical stresses on reconstruction plates. These stresses in association with the forces caused by contracture of the masticatory muscles during mastication causes strain, metal fatigue, and eventually plate fracture, which jeopardizes survival rate of the reconstruction plate and accordingly the success of reconstructive surgery [14]. This mechanical risk factor could justify plate fracture that happened to two of our patients $(\mathrm{n} 2, \mathrm{n} 15)$ who encountered resection of the mandibular angle. Besides, our findings ascertained that plate fractures are significantly related to mandibular angle defects.

Furthermore, Arias-Gallo [15] reported that most hardware exposure occurs at sites exposed to higher moment and shear forces. These factors are even exaggerated by the excessive intraoperative bending of titanium reconstruction plates [16]. In the current study, one of our patients (n17) experienced plate exposure at the premolar region owing to plate overbending.

Radiotherapy is a biological risk factor that leads to plate-related complications. Our findings that RT is significantly associated with plate exposure are consistent with the reports of Ryu [17]. He explained that ionizing radiation causes damage to the small vessels and progressively occlusion and fibrosis of the vessels. This decreases the local tissue vascularity and alteration of the bone-to-metal interface. The poor soft tissue bed causes infection and fistula formation that leads to plate exposure.

In the light of the previous explanation, two patients (n9, n11) developed orocutaneous fistulas and plate exposure. These patients were treated for squamous cell carcinoma and received postoperative radiotherapy. The condition necessitated plate removal and application of external pin fixation together with culture and sensitivity tests to control the infection. The treatment with external pin fixation decreased the tension over the soft tissue by avoiding the bulky hardware underneath the mucosa and thus enhanced the healing.

In addition, Ryu [17] stated that radiotherapy causes back-scatter effect against the titanium plates that increases the risk of local overdoses around the plate and contribute to screw loosening. This explains why screw loosening occurred in case n19 who was receiving ionizing radiation. On the other hand, loosening of the screws occurred in case $\mathrm{n} 13$ who was not receiving RT. Our explanation is consistent with that of Kimura et al. [18], who explained this as a result of the masticatory forces that leads to vertical stress on the plate leading to bone resorption around the screws.

Another complication was heat sensitivity, which was encountered in one patient (n8) owing to the extension of the reconstruction plate anteriorly crossing the midline. Our results are in agreement with Cavuşoğlu et al. [19], who reported that some patients might experience cold or heat sensation especially at the anterior region where the mucosa is thin, but the condition soon resolved after about 3 months as the patient can put up to the complication.

\section{CONCLUSION}

The mandibular reconstruction plate's survival rate is significantly related to mechanical and biological risk factors in terms of location of mandibular defects and RT, respectively, and was not significantly related to the age or sex of the patients.

The mandibular reconstruction plate survival rate is significantly related to biological risk factors, such as location of mandibular defects, RT, and mechanical risk factors.

\section{RECOMMENDATIONS}

1. Under-contouring of reconstruction plates is a good approach to avoid future plate exposure because of plate over-bending.

2. External pin fixation could be a useful tool to maintain the mandibular space in irradiated patients after the removal of the reconstruction plate, but on the other hand it is cumbersome to the patient's quality of life.

3. Nowadays, sterolithiography models are very promising tools in decreasing the drawbacks of the conventional reconstruction plates.

\section{CONFLICT OF INTEREST}

There are no conflicts of interest.

\section{REFERENCES}

1. Schmeizeisen R, Schön R. Microvascular reanastomozed allogenous iliac crest transplants for the reconstruction of bony defects of the mandible in miniature pigs. Int J Oral Maxillofac Surg 1998; 27:377-385. 
2. Anthony J. Donor leg morbidity and function after fibula free flap mandible reconstruction. Plast Reconstr Surg 1995; 96:146-152.

3. Boyd J. Use of reconstruction plates in conjunction with soft-tissue free flaps for oromandibular reconstruction. Clin Plast Surg 1994; 21:69-77.

4. Urken M. Oromandibular reconstruction using microvascular composite flaps: report of 210 cases. Arch Otolaryngol Head Neck Surg 1998; 124:46-55.

5. Maurer P, Eckert AW, Kriwalsky MS, Schubert J. Scope and limitations of methods of mandibular reconstruction: a long-term follow-up. Br J Oral Maxillofac Surg 2010; 48:100-104.

6. Peacock ZS, Afshar S, Lukas SJ, Kaban LB. Customized repair of fractured mandibular reconstruction plates. J Oral Maxillofac Surg 2012; 70: e563-e573.

7. Knott P. Evaluation of hardware-related complications in vascularized bone grafts with locking mandibular reconstruction plate fixation. Arch Otolaryngol Head Neck Surg 2007; 133:1302-1306.

8. Szypryt P. The use and abuse of locking plates. Orthop Trauma 2009; 23:281-290.

9. Probst FA, Mast G, Ermer M, Gutwald $\mathrm{R}$, Schmelzeisen $\mathrm{R}$, Pautke $\mathrm{C}$, et al. MatrixMANDIBLE preformed reconstruction plates - a two-year twoinstitution experience in 71 patients. J Oral Maxillofac Surg 2012; 70: e657e666.

10. Cohen A, Laviv A, Berman $P$, Nashef R, Abu-Tair J. Mandibular reconstruction using stereolithographic 3-dimensional printing modeling technology. Oral Surg Oral Med Oral Pathol Oral Radiol Endod 2009; 108:661-666.
11. Jewer D. Orofacial and mandibular reconstruction with the iliac crest free flap: a review of 60 cases and a new method of classification. Plast Reconstr Surg 1989; 84:391-403.

12. Seol G-J. Reconstruction plates used in the surgery for mandibular discontinuity defect. J Korean Assoc Oral Maxillofac Surg 2014; 40:266-271.

13. Shibahara T. Fracture of mandibular reconstruction plates used after tumor resection. J Oral Maxillofac Surg 2002; 60:182-185.

14. Nagasao T, Miyamoto J, Tamaki T, Kawana H. A comparison of stresses in implantation for grafted and plate-and-screw mandible reconstruction. Oral Surg Oral Med Oral Pathol Oral Radiol Endod 2010; 109:346-356.

15. Arias-Gallo J. Long term results of reconstruction plates in lateral mandibular defects. Revision of nine cases. Auris Nasus Larynx 2004; 31:57-63.

16. Knoll W-D. Analysis of mechanical stress in reconstruction plates for bridging mandibular angle defects. J Craniomaxillofac Surg 2006; 34 : 201-209.

17. Ryu J. Mandibular reconstruction using a titanium plate: the impact of radiation therapy on plate preservation. Int J Radiat Oncol Biol Phys 1995; 32:627-634.

18. Kimura A, Nagasao $\mathrm{T}$, Kaneko $\mathrm{T}$, Tamaki $\mathrm{T}$, Miyamoto J, Nakajima T. Adequate fixation of plates for stability during mandibular reconstruction. J Craniomaxillofac Surg 2006; 34:193-200.

19. Cavuşoğlu T. Resorbable plate-screw systems: clinical applications. Ulus Travma Acil Cerrahi Derg 2005; 11:43-48. 